\title{
Interaction between the Arterial Baroreflex and a Hypertensive Stressor: a Mathematical Model
}

\author{
MC Melgers, CA Swenne, H van de Vooren, EE van der Wall \\ Leiden University Medical Center, Leiden, The Netherlands
}

\begin{abstract}
Stressors intensify sympathetic outflow, thus increasing heart rate and arterial blood pressure. The significance of the arterial baroreflex is to be found in reducing such rate and pressure changes.

For a better understanding of the interaction between a hypertensive stressor and the arterial baroreflex we have developed a mathematical simulation model, consisting of a hemodynamic section, a baroreceptor section, and a baroreflex section. Physiological or pathological resting conditions are simulated by specific settings of the autonomic tone parameters and cardiac stroke volume. Also the vagal and sympathetic baroreflex gains can be set. A stressor is introduced by adding extra sympathetic outflow to the heart and the peripheral resistance. The responses in heart rate and blood pressure help to understand how the arterial baroreflex protects the heart and the circulation.
\end{abstract}

\section{Introduction}

Many stressors intensify sympathetic outflow, thus increasing heart rate and arterial blood pressure. In this context, the clinical significance of the arterial baroreflex is to be found in reducing such rate and pressure changes. This would limit cardiac oxygen consumption (which is, in first approximation, proportional to the product of heart rate and systolic blood pressure) and reduce the risk of vascular ruptures. Baroreflex protection is restricted in time as baroreceptors reset after 10-30 minutes. However, as many daily life stressors have a relative short duration (minutes) and there is no other quick acting control mechanism that the body can recruit, baroreflex circulatory control remains a protective mechanism of primary importance.

It has been demonstrated that baroreflex sensitivity has independent predictive value in the setting of myocardial infarction and chronic heart failure. The mechanisms via which the baroreflex exerts its beneficial influence have, however, poorly been studied. We presume that the interaction of the baroreflex with stressors of various nature is pertinent here, and use a mathematical model to get more insight in this process. The study presented here describes the model and the first simulation results.

\section{Methods}

Like in our previous study, in which we investigated the baroreflex transfer function [1], the model for the current study was based on the one developed by TenVoorde [2]. An outline of our model - like the original one implemented in Matlab Simulink - is given in Figure 1. It has a beat-to-beat hemodynamic part (Starling heart, Windkessel) and a continuous neural part with different dynamics for the sympathetic and vagal branches.

At the baroreceptors, the systolic blood pressure (SBP) is compared with a reference value (the low-pass filtered systolic blood pressure, the filter representing continuous baroreceptor resetting). After a delay, the autonomic nervous system reacts by modulating the autonomic resting tones to he heart (vagal and sympathetic) and to the peripheral resistance (sympathetic), the modulating signals being proportional to the difference between the actual blood pressure and the reference value. The sinus node then generates a time varying heart rate (HR) according to the Rosenblueth-Simeone equation. This is essentially a multiplication of the intrinsic heart rate, an index $\mathrm{m}$, representing sympathetic tone, and an index $\mathrm{n}$, representing vagal tone. When sympathetic tone increases, $m$ increases too; by defnition $m>1$. When vagal tone increases, $\mathrm{n}$ decreases, by definition $\mathrm{n}<1$.

Following rate generation, instantaneous heart rate is converted into interbeat intervals (IBI). The Starling heart fills throughout the interbeat interval: a larger IBI value causes a larger stroke volume. The reference volume, $\mathrm{V}_{\text {ref, }}$ denotes the stroke volume with a 1-second filling time). Stroke volume determines the Starling heart's pulse pressure. Diastolic blood pressure is controlled by the Windkessel time constant (under influence of the dynamically changing peripheral resistance), by IBI, and by the pulse pressure. Finally, the systolic blood pressure (SBP) is computed by adding diastolic blood pressure and pulse pressure. 


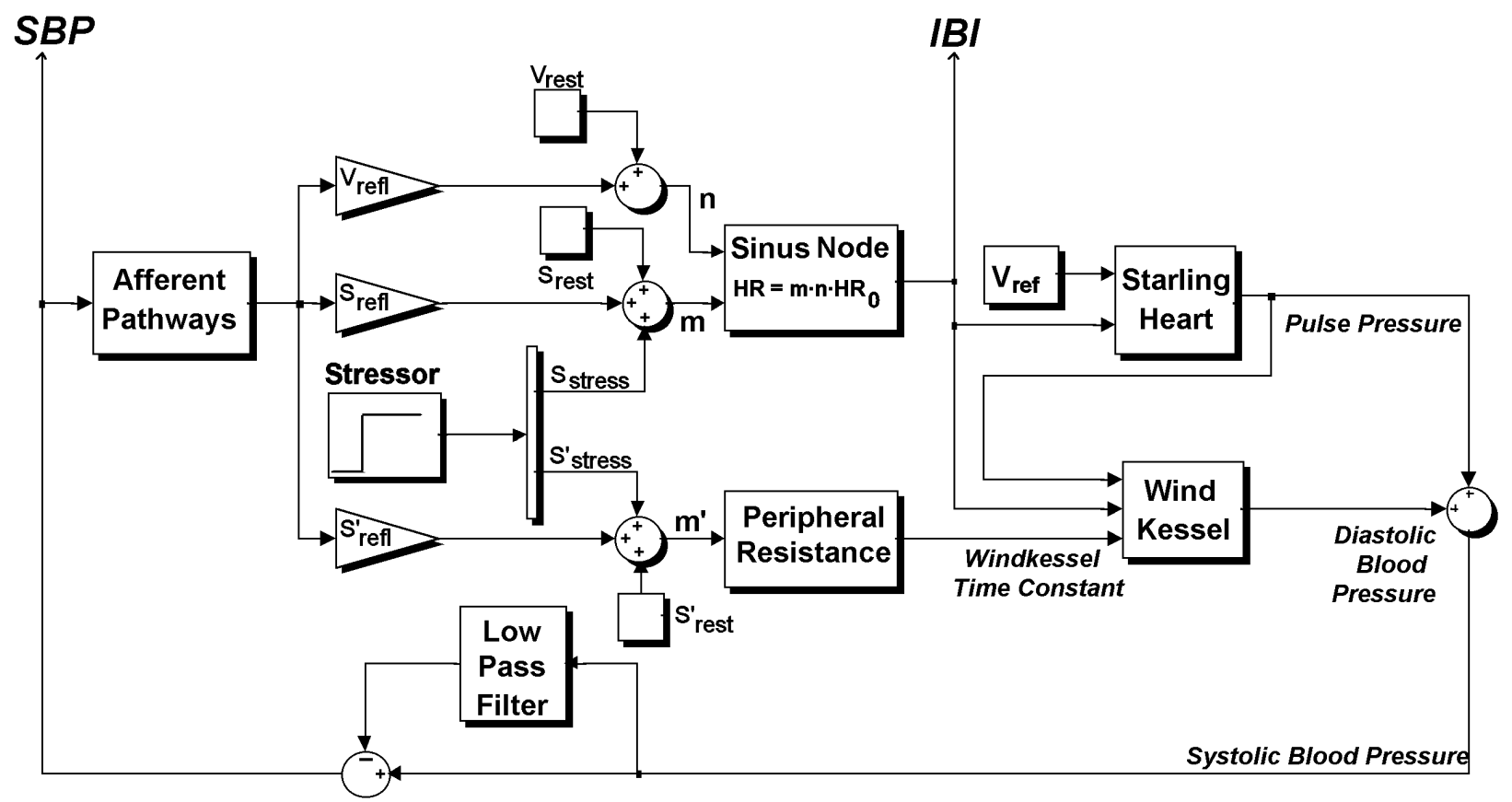

Figure 1: Simulation model - see text for description. $V_{\text {refl }}, S_{\text {refl }}, S_{\text {reff }}$ : baroreflex gains (see Table 1); $V_{\text {rest }}, S_{\text {rest }}, S_{\text {rest }}$ : autonomic resting tones; $S_{\text {stress }}, S^{\prime}{ }_{\text {stress }}$ : balanced stressor output; $V_{\text {ref }}$ : reference stroke volume; $m$, m', n: autonomic outflow.

A stressor was modelled by adding an extra amount of sympathetic outflow to the heart and to the peripheral resistance. This is done with an adjustable balance, as the distribution of a stressor over the heart and the peripheral resistance is not known. No matter the balance setting (all stress on the heart, all stress on the peripheral resistance, or a mix of both) we adjusted the amount of stress in such a way that in absence of baroreflex control (all three gains set to 0) the product of heart rate and systolic blood pressure increased by $25 \%$.

A physiological and a pathophysiological situation was modelled by different settings of the vagal and sympathetic resting tones and of the reference stroke volume of the Starling heart. Healthy subjects ('Normals') were simulated by setting the reference stroke volume at $80 \mathrm{ml}$, and the resting tones at 1.2 (sympathetic tone to the heart), 0.5 (vagal tone to the heart) and 1.2 (sympathetic tone to the peripheral resistance). In this way, $\mathrm{HR}$ and $\mathrm{SBP}$ generated by the model are $60 \mathrm{bpm}$ and $119 \mathrm{mmHg}$, respectively. Diseased subjects ('Patients') were simulated by setting the reference stroke volume at $60 \mathrm{ml}$, and the resting tones at 1.5 (sympathetic tone to the heart), 0.6 (vagal tone to the heart) and 1.25 (sympathetic tone to the peripheral resistance). In this way, $\mathrm{HR}$ and SBP generated by the model are $90 \mathrm{bpm}$ and $113 \mathrm{mmHg}$, respectively. We made this choice because in severe chronic heart failure hearts are often protected by adjusting the medication until a relatively low systolic blood pressure is achieved.
Simulations were done for normals and for patients with varying settings of the stressor balance, thus covering the range from the application of all stress to the heart, via a mixture of stress opposed to the heart and to the peripheral resistance, to unique stressing via the peripheral resistance.

For each of the three limbs of the baroreflex (sympathetic to the heart, vagal to the heart, and sympathetic to the peripheral resistance) we defined, in addition to the standard gain in the original ten Voorde model [2], a low and a high gain (0.3 and 3.0 times the standard gain, respectively). Simulations were done for 9 of the 27 possible combinations of a weak, normal, or high gain in the sympathetic branch to the heart, the vagal branch to the heart, and the sympathetic branch to the peripheral resistance (see Table 1). Each of these combinations is associated with a BRS value, these values were measured in a previous study by means of simulations [1]. It has to be kept in mind here that BRS characterizes baroreflex control of the heart, thus leaving an essential part of its function - control of the peripheral resistance - out of consideration.

Every run consisted of 3 minutes simulation of the resting state followed by 3 minutes simulation of the stressed state, at the end of which we determined the simulation results. The 3-minute periods were chosen to allow the model to converge to a stationary state ( in practice reached within 1 minute). 

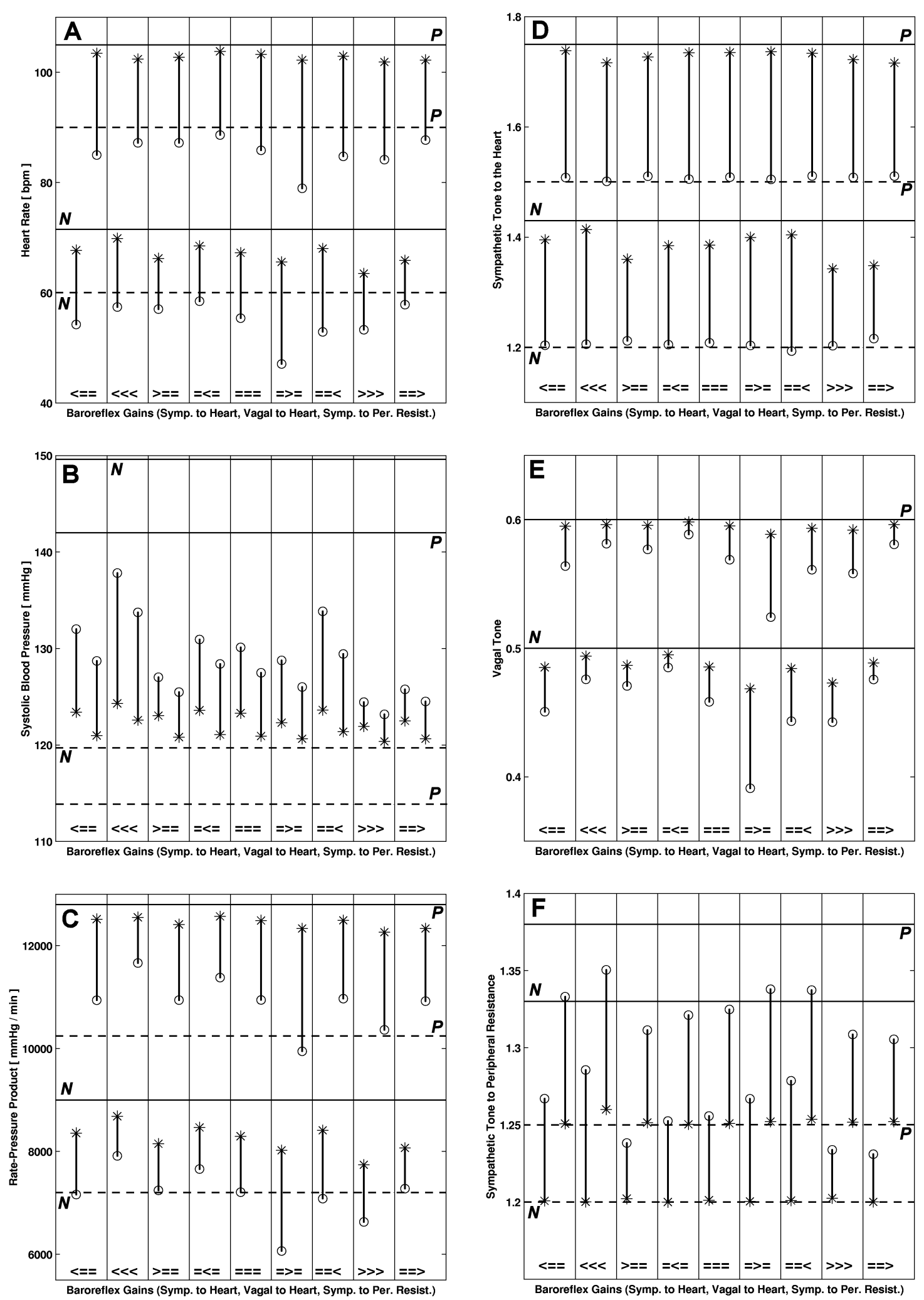

Figure 2: Simulation results, see text for description. 


\begin{tabular}{|c|c|c|c|c|}
\hline \multicolumn{3}{|c|}{ Baroreflex gains } & \multirow{2}{*}{$\begin{array}{l}\text { Normals } \\
\text { BRS } \\
{[\mathrm{mmHg}]}\end{array}$} & \multirow{2}{*}{$\begin{array}{l}\text { Patients } \\
\text { BRS } \\
{[\mathrm{mmHg}]}\end{array}$} \\
\hline $\begin{array}{l}\text { Symp. } \\
\text { (Heart) }\end{array}$ & $\begin{array}{l}\text { Vagal } \\
\text { (Heart) }\end{array}$ & $\begin{array}{l}\text { Symp. } \\
\text { (Per.Res.) }\end{array}$ & & \\
\hline \multicolumn{3}{|c|}{ partial $\beta$-adrenergic blockade } & \multirow{2}{*}{7.80} & \multirow{2}{*}{4.65} \\
\hline 0.33 & \begin{tabular}{l|l|} 
& 1.0
\end{tabular} & 1.0 & & \\
\hline \multicolumn{3}{|c|}{ weak baroreflex } & \multirow{2}{*}{2.70} & \multirow{2}{*}{1.76} \\
\hline 0.33 & \begin{tabular}{l|l} 
& 0.33 \\
\end{tabular} & 0.33 & & \\
\hline \multicolumn{3}{|c|}{ cardiac symp. predominance } & \multirow{2}{*}{5.83} & \multirow{2}{*}{3.37} \\
\hline 3.0 & \begin{tabular}{|l|l|} 
& 1.0
\end{tabular} & 1.0 & & \\
\hline \multicolumn{3}{|c|}{ partial cholinergic blockade } & \multirow{2}{*}{2.09} & \multirow{2}{*}{1.28} \\
\hline 1.0 & \begin{tabular}{l|l} 
& 0.33 \\
\end{tabular} & \begin{tabular}{l|l} 
& 1.0 \\
\end{tabular} & & \\
\hline \multicolumn{3}{|c|}{ normal baroreflex } & \multirow{2}{*}{7.22} & \multirow{2}{*}{4.07} \\
\hline 1.0 & \begin{tabular}{l|l|} 
& 1.0 \\
\end{tabular} & 1.0 & & \\
\hline \multicolumn{3}{|c|}{ parasymp. predominance } & \multirow{2}{*}{22.99} & \multirow{2}{*}{12.96} \\
\hline 1.0 & \begin{tabular}{l|l} 
& 3.0 \\
\end{tabular} & 1.0 & & \\
\hline \multicolumn{3}{|c|}{ partial $\alpha$-adrenergic blockade } & \multirow{2}{*}{7.22} & \multirow{2}{*}{4.07} \\
\hline 1.0 & \begin{tabular}{l|l|} 
& 1.0
\end{tabular} & 0.33 & & \\
\hline \multicolumn{3}{|c|}{ strong baroreflex } & \multirow{2}{*}{21.15} & \multirow{2}{*}{11.98} \\
\hline 3.0 & \begin{tabular}{l|l|} 
& 3.0 \\
\end{tabular} & 3.0 & & \\
\hline \multicolumn{3}{|c|}{$\begin{array}{l}\text { symp. predominance to per. } \\
\text { resistance }\end{array}$} & \multirow{2}{*}{7.22} & \multirow{2}{*}{4.07} \\
\hline 1.0 & 1.0 & 3.0 & & \\
\hline
\end{tabular}

Table 1: Baroreflex gain combinations for which simulations were run. The combinations are listed in the order in which they appear in every panel of Figure 2.

\section{Results}

Figure 2 shows the simulation results for heart rate (panel A), systolic blood pressure (B), rate-pressure product $(\mathrm{C})$, sympathetic tone, $\mathrm{m}$, to the heart $(\mathrm{D})$, vagal tone, n, (E) and sympathetic tone, m', to the peripheral resistance (F). Dashed horizontal lines marked with $\mathrm{N}$ and $\mathrm{P}$ give the resting values for normals and patients; solid horizontal lines marked with $\mathrm{N}$ and $\mathrm{P}$ denote the values under stress when the baroreflex is inactivated (all gains $\mathrm{V}_{\text {refl }}, \mathrm{S}_{\text {refl }}, \mathrm{S}_{\text {refl }}=0$, rate-pressure product increase $=$ $25 \%$; Figure 3 ). Trajectories between asterisks and open circles represent results with a stressor balance ranging between all stress opposed to the heart and all stress opposed to the peripheral resistance. The nine panels marked with ' $<==,, \ldots$, ' $==>$ ' depict results obtained with the nine different combinations of baroreflex gains listed in Table 1. For example, the combination ' $<=>$ ' denotes the values $0.33,1.0$ and 3.0. For every combination the left trajectory represents a normal subject, while the right trajectory represents a patient.

\section{Discussion}

Panel A in Figure 2 shows that heart rate reduction is limited when the stressor is fully applied to the heart. However when fully applied to the peripheral resistance, reflex vagal activation may elicit a rate reduction even till under the resting level. Both extremes may not be realistic, though we have seen handgrip responses where blood pressure, but not heart rate, increases (unpublished). With mental stress in normals we have even observed heart rate to slightly decrease [3].

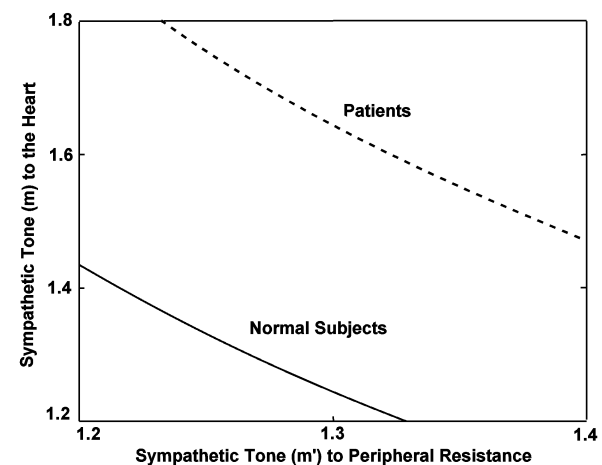

Figure 3. Combinations of sympathetic tones to the heart and to the peripheral resistance that generate a $25 \%$ ratepressure product increse with inactivated baroreflex.

Panel B in Figure 2 shows that there is little blood pressure increase when the stressor is opposed to the heart. It also shows that even a weak baroreflex may be important for blood pressure reduction. The association between the BRS values as listed in Table 1 and the reduction in blood pressure is not impressive, this is due to the fact that BRS values do not characterize baroreflex control of the peripheral resistance. Panel $\mathrm{C}$ shows that when the stressor is applied to the heart, the reduction of the rate-pressure product is not very impressive, while baroreflex buffering is much more efficient for a stressor applied to the peripheral resistance.

In conclusion, the model presented here is helpful in understanding the efficacy of the arterial baroreflex. In order to make the model applicable in clinical practice we are currently comparing real-life and simulated data.

\section{References}

[1] Van de Vooren H, Swenne CA, Ten Voorde BJ, Van der Wall EE. Assessment of Baroreflex Sensitivity by the Closed-Loop Blood Pressure to Interbeat Interval Transfer Function. Computers in Cardiology 2001;28:489-492.

[2] Ten Voorde BJ, Kingma R. A baroreflex model of short term blood pressure and heart rate variability. Stud Health Technol Inform 2000;71:179-200.

[3] Swenne CA, Bootsma M, Van Bolhuis HH. Different autonomic responses to orthostatic and to mental stress in young normals. Homeostasis 1995;36:287-292.

Address for correspondence.

Cees A. Swenne, PhD

Cardiology Department, Leiden University Medical Center

PO BOX 9600, 2300 RC Leiden, The Netherlands

Phone: +31-71-526-1972, Fax: +31-71-526-6809

E-mail: c.a.swenne@lumc.nl 